\title{
Análisis de la transferencia de la expresión escrita desde discursos posterior a aprendizajes en cursos formativos
}

\author{
Analysis of the transfer of written expression from the post-learning \\ speeches to training courses
}

\section{Dennis Lazo Ramos}

\section{Revista \\ Educación y Sociedad}

\section{Citar como: Lazo, D. (2021). Análisis de la transferencia de la expresión escrita desde discursos posterior a aprendizajes en cursos formativos. Revista Educación y Sociedad, 02(03), 17-28. doi: 10.53940/reys.v2i3.63}

Artículo recibido: 30-06-2021 Artículo aprobado: 22-07-2021 Arbitrado por pares

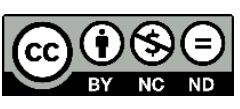

\section{ACEES}

\section{Resumen}

En el presente trabajo, se analiza el logro de la transferencia de la expresión escrita de alumnos de últimos ciclos en una universidad particular. Para lograr esto, nos hemos basado en teorías de gestión del conocimiento y teorías educativas constructivistas. Planteamos que una correcta evaluación de la expresión escrita solo se puede lograr si nos alejamos contextualmente y en contenido del aprendizaje primigenio. Esto solo se puede lograr si realizamos una evaluación de la competencia desde la transferencia. Por ello, el siguiente trabajo evalúa la redacción para identificar de qué modo han logrado transferir la competencia de expresión escrita desarrollada.

Palabras clave: transferencia, expresión escrita, evaluación

\begin{abstract}
In the present work, we analyze the achievement of the transfer of the written expression of students of last cycles in a particular university. We have relied on knowledge management theories and constructivist educational theories. We propose that a correct evaluation of a competence, in this case written expression, can only be achieved if we move away contextually and in content from the original learning took place. In that sense, this can be achieved if we develop an evaluation of the transfer. The following work assesses the writing of students to identify how they transfer the written expression competence.
\end{abstract}

Key words: transference, written expression, evaluation

\footnotetext{
1 Universidad Le Cordon Bleu (Perú). dlazor@pucp.edu.pe (iD) https://orcid.org/0000-0002-7324-8651

El presente artículo se desprende de la tesis para optar por el grado de Maestro en Educación con mención en Docencia e Investigación en Educación Superior por la Universidad Peruana Cayetano Heredia, titulada "Análisis de los procesos de transferencia de conocimientos de lenguaje en contextos de aprendizaje de cursos formativos".
} 


\section{Introducción}

Actualmente, existe una problemática muy marcada sobre cómo podemos analizar y comprender el logro o no de una competencia. Esto, principalmente, se debe a que, si bien se trabaja un enfoque por competencias, no se realiza, por lo general, una comprensión completa y holística de la evaluación de estos saberes, de modo que se termina optando por aplicar evaluaciones clásicas o que se concentran en el logro de un curso y no se enfocan en el desarrollo de la competencia en un nivel determinado. Esto se evidencia, en mayor medida, en el aprendizaje y evaluación de competencias generales. Entre estas, destaca la competencia de expresión escrita, ya que se ha establecido como constante un tipo de enseñanza o modelo por competencias que se caracteriza por estar centrado en el contexto cultural y social del estudiante o concentrarse en la carrera elegida por el estudiante (Cassany, 1990, Cassany, 2012 y Mostacero, 2006). Sin embargo, no se logra ir más allá del curso, ya que se genera un fetichismo por la forma y el producto en sí y esto no tiene una aplicación o posibilidades de uso y abstracción más allá del curso (Cisneros, Olave y Rojas, 2012; Aragón y Caicedo, 2009; Trillos, 2013). En ese sentido, existe una problemática no solo en cómo y qué se enseña, sino también en cómo se evalúa.

La situación mencionada previamente se debe a que la evaluación de aprendizajes, en la enseñanza convencional de la expresión escrita, se ha limitado a aplicar modelos. Si bien es cierto que la temática es libre, el proceso de redacción se encuentra aprisionado, de modo que no se produce una autonomía en el estudiante y no se permite que elabore productos verdaderamente propios. Este tipo de enseñanza imposibilita que lo aprendido se aprehenda en escenarios diferentes y, por ello, el saber queda recluido en el curso en sí y se vuelve impensable la posibilidad de potenciar la competencia en cursos posteriores. Debido a la situación explicada, evidenciamos varios problemas; sin embargo, el más destacable es el que implica la imposibilidad de identificar la problemática en sí y todo lo que acarrea. Lo acaecido se produce por un incorrecto proceso de evaluación por competencias y por una incorrecta evaluación y reelaboración de los modelos didácticos en general.

Una posibilidad que surge para resolver los problemas previamente indicados es realizar una evaluación de la competencia de la expresión escrita desde la transferencia. Esto se debe a que este constructo permite identificar el grado de profundidad que tiene un estudiante de abstraer un conocimiento y de reintegrarlo en un nuevo escenario. Esto es importante, ya que le permite traspasar diferentes tareas y logros de diversos cursos e integrarse a una futura profesionalización. En ese sentido, la transferencia o, mejor dicho, la evaluación de la transferencia funciona como modelo evaluativo mientras más alejados nos encontremos, a nivel temporal, contextual y cognitivo, del aprendizaje primigenio (Bloom, 2007).

Ahora bien, es necesario ver la activación de la transferencia, ya que determina el nivel de logro de la competencia y las posibilidades que tiene el estudiante de usarla en contextos posteriores y poco similares al del aprendizaje primigenio. Si esto se logra, estaríamos refiriéndonos a un aprendizaje significativo y para toda la vida.

El presente artículo se centra en una mirada de la transferencia como modelo evaluativo de la expresión escrita y, por ello, busca analizar textos de estudiantes de últimos ciclos de la carrera de administración de una universidad particular de Lima Metropolitana. Este producto, desde el análisis de contenido, se centra en la capacidad y posibilidades que tiene el estudiante para transferir lo aprendido en cursos generales de redacción en un escenario posterior y diferente al espacio de aprendizaje primigenio. Por ello, se busca conocer la viabilidad del modelo e identificar las posibilidades que tienen los estudiantes para reelaborar conocimientos que han aprendido en los primeros ciclos en cursos finales de su carrera. De este modo, podemos identificar qué aspectos han sido determinantes en su desarrollo y qué factores han imposibilitado el logro de la competencia a cabalidad desde la posibilidad para transferir. En ese sentido, entendemos el logro de una competencia como su posible aprehensión y 
desarrollo en escenarios diferentes y futuros vinculados al plano personal, académico y profesional del estudiante.

Por lo indicado anteriormente, este artículo busca evidenciar la necesidad de integrar el modelo de evaluación por competencias desde el logro de la transferencia para lograr que aquella sea más integral. Esto lo consideramos relevante, ya que el tipo de formas de evaluación, como veremos más adelante, resulta cíclico, pero en un sentido negativo: no se va más allá en la enseñanza y evaluación, de modo que se logra una competencia, pero que se queda estancada en el nivel de logro del curso.

\section{Modelos evaluativos de la expresión escrita}

Desde hace un tiempo, ha perdido relevancia la enseñanza del lenguaje más teórica y netamente gramatical o vinculada a preceptos. Se ha pasado a una enseñanza más práctica que busca que el aprendizaje se dé en un contexto identificable de manera vivencial para el estudiante, de modo que se active desde lo lúdico y desde la utilidad al proceso de redacción. En esa línea, tenemos propuestas que se centran en el desarrollo de textos relacionados con temas sociales, culturales que le importen al estudiante (Cassany, 1990, Cassany, 2012) o perspectivas más centradas en redacciones en relación con la carrera que está estudiando aquel, es decir, un modelo más profesionalizante desde el interés (Mostacero, 2006).

Ahora bien, el problema no es el modelo de enseñanza, sino su aplicación y la forma como se identifica o no el logro de la competencia, el cual se centra más en el logro del curso y no de aquella.

En ese sentido, las investigaciones realizadas en el marco de la expresión escrita y su evaluación pueden comprenderse desde tres espacios. En primer lugar, existe la intencionalidad de reforzar aspectos vinculados con la metacognición permite incrementar el nivel de redacción en los estudiantes (Cisneros, Olave y Rojas, 2012; Aragón y Caicedo, 2009; Trillos, 2013; Gatti, 2010; y Chirinos, 2012). En todos estos estudios, se consiguen resultados positivos a partir de la motivación de los estudiantes y reincidir en el proceso metacognitivo. Por ello, la función docente se constituye como la de un guía que posibilita que el estudiante interprete e identifique aquello que está logrando. El problema en estos trabajos radica en que los textos enseñados o de práctica tienen mucha similitud en extensión y características formales que el texto a evaluar, de modo que se puede afirmar que se trata de procesos aplicativos y no transferenciales o que buscan la aprehensión del conocimiento.

La segunda aproximación al fenómeno estudiado tiene muchas similitudes con el anterior apartado, salvo que se centran en una posición más existencial del alumno que redacta. Esto quiere decir que el sujeto, como ser arrojado al mundo, va a escribir o, en todo caso, tener mayor predisposición a escribir acerca de lo que le interesa; de lo que vivencia (Makuc, 2008; De Castro, 2006). En este tipo de postura, el estudiante es quien marca el ritmo, de modo que cuestiones teóricas no son necesarias; y lo que debe primar es que, mediante la práctica y el ensayo error, se llegue a un mayor nivel en la elaboración de los textos.

Además de lo previamente señalado, se evidencian vertientes que le brindan mayor preponderancia al rol del docente (Álvarez y Ramírez, 2012; Martínez, Díaz y Rodríguez, 2011). En ese sentido, se promueve un rol más activo y neutral del docente, ya que no solo es quien imparte conocimientos teóricos necesarios para el aprendizaje, sino es quien debe facilitar y guiar a los estudiantes al logro de sus redacciones.

De los antecedentes mencionados, podemos encontrar un aspecto en común a modo general, el cual es determinante para la indagación que estamos haciendo y para el modelo que estamos proponiendo: 
se desarrolla una enseñanza centrada en el estudiante desde el tema a elegir, hasta el rol que desempeña el docente; sin embargo, este posicionamiento es obviado al momento de la evaluación. Esta situación se ebe a que se establecen modelos muy similares que no retan al estudiante y que no permiten identificar hasta qué lugar puede llegar su razonamiento. Se generan evaluaciones similares a los textos prácticos, de modo que se impide que, dentro del curso, se hagan actos de desplazamiento. Este tipo de evaluación no solo no es retadora, cíclica y autorreferencial, sino que no va más allá del curso e impide conocer cómo y en qué nivel realmente se ha logrado la competencia y el grado de relación que esta tiene con otras, tanto de carácter general como específico. Por otro lado, un aspecto que impide comprender lo mencionado es la temporalidad: las evaluaciones son dentro del mismo curso. Nuevamente, lo que se ve es la competencia dentro del curso y no el desarrollo de esta. En ese sentido, no se evidencian evaluaciones posteriores al aprendizaje a nivel temporal ni a nivel de tipo de redacción solicitada. Para poder lograr esto, nos brindan grandes alcances la transferencia como modelo evaluativo; aspecto que elaboraremos a continuación.

\section{Aspectos teóricos para una evaluación integral de la expresión escrita}

\section{Procesos de aprendizaje del lenguaje}

Partiendo de Piaget (2001) comprendemos el aprendizaje como una construcción repleta de complejidades que se caracterizan, entre otras cosas, por la asimilación y la reelaboración u organización de lo aprendido por el sujeto para nuevos contexto o situaciones diarias que implican diversos niveles cognitivos. En línea con esto, el aprendizaje significativo (Ausubel, Novak y Hanesian, 1983) implica una reingeniería del estudiante del saber recibido que consiste, necesariamente, en una aprehensión compleja. Esto solo se logra al integrar, al aprendizaje nuevo, vivencias pasadas y una enciclopedia cultural, social y personal que posee el alumno.

Lo indicado anteriormente no se ha elaborado en lo concerniente al aprendizaje del lenguaje desde la filosofía del lenguaje, salvo, de manera incipiente, por Van Dijk y Kintsch (1983), quienes priorizan los conocimientos previos del lector para realizar nuevos textos y enmarcan múltiples niveles para que se dé una comprensión de textos fundamental para poder redactor. Para solucionar esta situación, el paso más lógico es retornar a ciertos supuestos elaborados desde la filosofía del lenguaje (Wittgenstein, 1998) y desde la pragmática (Austin, 1998). De lo propuesto por estos autores, y comparándolo con lo mencionado previamente sobre la practicidad de la lengua y su enseñanza, podemos afirmar que resulta necesario un aprendizaje teórico que, paradójicamente, establezca parámetros y niveles en el desarrollo del estudiante, de modo que, ahí sí se posea una finalidad práctica. Ante esto, el logro de la expresión escrita está supeditada a una situación neutral que se puede vislumbrar de mejor manera desde la transferencia.

\section{La transferencia del aprendizaje como modelo evaluativo de la expresión escrita}

Cuando nos referimos a transferencia de la expresión escrita, debemos asir dos términos que no han sido investigados ni teorizados a cabalidad. A su vez, debemos vincular las concepciones que se tienen de transferencia desde la teoría educativa constructivista y la teoría del comportamiento organizacional. En ese sentido, deseamos mostrar un mapeo teórico para una futura triangulación de este con los resultados de la investigación.

En primer lugar, en lo relacionado con la transferencia desde la gestión y alta dirección, debemos indicar que es una tendencia que surge en los años noventa con la intencionalidad de poder comprender por qué la industria japonesa rendía mejor que la estadunidense. A partir de diversas investigaciones, se observa la existencia de un vacío entre aprendizaje teórico, el aprendido en el plano netamente académico y aprendizaje práctico, aprendido en el espacio profesional. (Szulanski, 1996, y Von Hippel, 
1994). De acuerdo con los primeros teóricos sobre esto, la formación ideal debe incluir un diálogo directo; un continuo entre aprendizaje explícito (el teórico) y el tácito (el práctico o empírico). Si no existe una retroalimentación y socialización constante entre ambos, solo podrá existir o una ejecución técnica sin relevancia analítica y, por ende, no creativa ni innovadora; o surgirá un escenario en extremo teórico y reflexivo, pero para nada práctico, lo cual implica que no podrá tener un impacto en la realidad. (Nonaka y Takeuchi, 1995). Ahora bien, ese quiebre indicado anteriormente es el denominado stickiness (Von Hippel, 1994), el cual solo se puede superar al realizar la retroalimentación.

Por otro lado, en el contexto educativo, surge una reinterpretación constructivista, la cual no dialoga con la teoría educativa. Esta es implementada, en diferentes niveles, por Carpintero y Beltrán (2010) y Bloom, J. (2007). Mientras que la primera lo observa de un modo más mecánico; el segundo lo ve como un proceso más analítico. Lo que tienen en común es que, partiendo de la idea de la transferencia como un constructo de alta complejidad (usando la taxonomía de Bloom), observan que este proceso es el ideal para realizar una evaluación por competencias completa y complejizada, la cual posibilita entender a cabalidad si se ha desarrollado o no una competencia y permite comprender el proceso como uno que va más allá de aprendizajes monolíticos que se realizan por cursos y no por el desarrollo integral y holístico de una competencia.

En el marco indicado en párrafos anteriores, surge la posibilidad de no solo desarrollar el proceso de transferencia en constructos complejos, sino en habilidades o actitudes, lo cual también denota complejidad. Collins (2012) lo realiza al observar la posibilidad de evaluar el proceso de transferencia de inteligencia emocional. Esto es posible, ya que, según él, la transferencia de actitudes se puede dar porque estas pueden ser socialmente aprendidas y desarrolladas en espacios de educación por medio de procesos vinculares. Aspecto similar es abordado por Buissink, Mann y Shephard (2011) al indicar que el proceso se puede hacer de la misma forma en lo relacionado a las actitudes que potencian y posibilitan que se desarrolle el dominio afectivo. Este proceso es desarrollado desde la teoría de Bloom, J. (2007), quien indica que la evaluación se puede realizar mientras más alejado sea contextualmente y en contenido del proceso primigenio de aprendizaje. Situación similar ocurre para la transferencia de competencias transversales (Arias y Collazos, 2015, y Bachand, 2011). El momento mencionado se puede desarrollar en competencias como pensamiento crítico (Maynard, 1991), expresión escrita (Lazo, 2017) y pensamiento creativo (Badea, Prostean, Adam y Giuca, 2013). Lo explicado previamente, sea desde una teoría educativa, del comportamiento organizacional o ambas, puede desarrollarse por la naturaleza compleja de una competencia transversal: no queda solo en el curso, sino se va desarrollando a lo largo de la carrera y vida profesional del alumno, de modo que debe potenciarse por diferentes parámetros y, por dicha complejidad, el modo más efectivo de poder comprender si se desarrolla es desde la transferencia.

Ahora bien, en lo relacionado a la evaluación de la transferencia, a nivel teórico, como habíamos indicado anteriormente, esta solo se puede desarrollar ante la ruptura de lo teórico con lo práctico. No se produce por un vacío entre teoría y práctica (Crochet, Agostini, Knight, Resseguier, Berdah y Aggarwal, 2017; Alt-Gehrman, 2019; y Becheikh, Ziam, Idrissi, Castonguay y Landry, 2010). En ese sentido, Blackman y Benson (2010) proponen que es necesario complementar aprendizajes para erradicar dicha ruptura, de modo que solo así se logre un aprendizaje por competencias significativo. En relación con esto, teorías actuales siguen manteniendo la noción de que solo se puede lograr si se integra el aprendizaje tácito con el explícito, de modo que se convierta en un proceso natural, completo, integral y, especialmente, cíclico (Nonaka y Takeuchi, 1995; Szulanski, 1996; y Von Hippel, 1994).

Si lo mencionado sobre la transferencia lo relacionamos con lo planteado desde la filosofía del lenguaje, veremos una constante interesante: los engranajes que impiden la transición; que generan este vacío que impide la transferencia, están supeditados a la necesidad de comunicarse y al interés por emitir una idea que sea entendida y asumida por otro. En ese sentido, los factores de interés y necesidad son 
determinantes para que exista una transferencia de la expresión escrita. Es decir, el interés y necesidad son ejes focales que permiten al alumno realizar la transición entre lo explícito y lo tácito; entre lo teórico y lo práctico y, de este modo, evitar el stickiness.

\section{Metodología}

El estudio realizado es de tipo cualitativo descriptivo. Se optó por analizar las redacciones de alumnos de noveno ciclo de la carrera de Administración de Negocios Hoteleros y Turísticos matriculados en el curso Metodología de Investigación. En ese sentido, el objetivo de la investigación es en analizar cómo y en qué nivel se ha cimentado lo aprendido en los primeros ciclos con los cursos de redacción, de modo que los alumnos puedan transferir lo aprendido en un contexto posterior y elaborando textos que no se relacionan con lo aprendido años atrás. Para que este proceso fuera más orgánico, se analizaron las redacciones que el mismo curso pedía: proyecto de investigación como posible tema de tesis.

Ahora bien, la razón por la que se decidió realizar un estudio cualitativo es por la complejidad para asir e interpretar los procesos de transferencia que se hayan realizado. Por ello, el desarrollo de este tipo de estudio nos permite analizar de modo complejo los textos parciales y finales de una cantidad pequeña de estudiantes desde el análisis de contenido. En ese sentido, para evaluar los procesos de transferencia de la competencia expresión escrita se desarrolló un diseño cualitativo hermenéutico. Por otro lado, de modo retrospectivo, comprenderemos en qué nivel y por qué motivos el alumno consigue o no reelaborar lo aprendido en cursos pasados y reformularlo para cumplir con un producto solicitado en un curso posterior. Ahora bien, hemos tomado este camino, ya que, de este modo, es posible entender de forma intrínseca los textos producidos y, así, evitamos incluir aspectos sociales o culturales tanto del alumno como del investigador.

En lo relacionado a la población, se trató de 15 estudiantes de noveno ciclo matriculados en el curso Administración de Negocios Hoteleros y Turísticos de una universidad privada de Lima Metropolitana. Todos estos alumnos conforman la promoción de esa carrera, ya que solo se abre un aula en ese ciclo y se trata de un curso obligatorio, previo a los seminarios de tesis. Además, todos son mayores de edad y sus edades oscilan entre los 21 y 25 años. Finalmente, es necesario mencionar que, como se va a evaluar, colateralmente, el proceso en su redacción, el único criterio de exclusión es que no hayan presentado las dos versiones del trabajo (avance parcial y trabajo final). Las dos redacciones están enmarcadas en el logro de la futura tesis. En ese sentido, los alumnos deben elaborar un proyecto de investigación. En el avance se elabora el planteamiento del problema, la justificación de este y los objetivos. Por otro lado, la versión final mejora todo lo avanzado y agrega la parte metodológica. Optamos por revisar y analizar los textos colateralmente a la revisión del docente para mantener su autonomía y el normal desarrollo del curso. Este último aspecto es considerado como necesario, ya que de este modo obtuvimos resultados más certeros y correspondientes con la realidad. Tanto el docente como los alumnos sabían y accedieron a la evaluación, pero también conocían que esto no tenía ninguna implicancia académica y profesional en su rol de estudiantes y docente.

Tras aplicar el único criterio de exclusión, quedaron 6 informantes con 2 textos cada uno. Esto se debió a que algunos se retiraron del curso y otros no entregaron una de las dos redacciones. A su vez, las únicas piezas de información fueron las dos redacciones de los alumnos. La temática fue elegida por ellos, pero enmarcada en su futura tesis de grado. El recojo de los textos y el análisis de la información, para una mayor comodidad de los alumnos y el docente y para no interferir con el transcurrir normal del curso, se realizó una vez calificados los trabajos. A su vez, tras la culminación del ciclo académico, se procedió a la codificación y análisis de resultados. 
Para el análisis de la información en relación con el logro o no de la transferencia de la expresión escrita en un escenario posterior a nivel temporal y temático, se usó como técnica el análisis de contenido. Asimismo, esto se hizo mediante la Guía para el análisis de la transferencia de expresión escrita, la cual es de elaboración propia, tras una validación con expertos usando el método Delphi. Esta guía está agregada como anexo, pero surgió de una operacionalización que mostramos a continuación:

Tabla 1

Operacionalización de la transferencia de conocimientos de redacción

Categoría: Transferencia de conocimientos de redacción en un contexto posterior temporal y temáticamente diferente

Definición conceptual:

Capacidad para percibir y evidenciar un conocimiento, y aplicarlo y modificarlo en contextos diferentes (Szulanski: 1996)

\begin{tabular}{|c|c|c|}
\hline $\begin{array}{l}\text { Definición } \\
\text { operacional }\end{array}$ & Categorías & Subcategorías \\
\hline \multirow{4}{*}{$\begin{array}{l}\text { Capacidad para } \\
\text { comprender y } \\
\text { evaluar una } \\
\text { situación } \\
\text { comunicativa y, } \\
\text { en base a eso, } \\
\text { reformular y } \\
\text { seleccionar } \\
\text { conocimientos } \\
\text { gramaticales y de } \\
\text { contenido para la } \\
\text { redacción de un } \\
\text { texto académico }\end{array}$} & $\begin{array}{l}1.1 . \\
\text { Interiorización y } \\
\text { aplicación de } \\
\text { conocimientos } \\
\text { teóricos } \\
\text { aprendidos en } \\
\text { CPL1 }\end{array}$ & $\begin{array}{l}\text { 1.1.1. Evalúa la redacción desarrollada para que no se } \\
\text { presenten errores de puntuación enseñados en CPL1 en } \\
\text { el texto. } \\
\text { 1.1.2. Ejecuta una redacción que contiene conectores y } \\
\text { uso de referentes enseñados en CPL1 } \\
\text { 1.1.3. Desarrolla el texto de una manera ordenada. } \\
\text { 1.1.4. Estructura el texto por medio de una adecuada } \\
\text { jerarquización de ideas }\end{array}$ \\
\hline & $\begin{array}{l}1.2 . \\
\text { Interiorización, } \\
\text { absorción y } \\
\text { desarrollo de } \\
\text { conocimientos } \\
\text { prácticos } \\
\text { aprendidos en } \\
\text { CPL1 }\end{array}$ & $\begin{array}{l}\text { 1.2.1. Selecciona las estrategias explicativas: } \\
\text { definición, aclaración, ejemplificación y causalidad } \\
\text { para el desarrollo de la redacción/exposición } \\
\text { 1.2.2. Aplica, cuando es pertinente, las estrategias } \\
\text { explicativas } \\
\text { 1.2.3. Se percibe una coherencia constante en el texto } \\
\text { gracias a una comprensión de las fuentes leídas en el } \\
\text { curso Seminario de Investigación }\end{array}$ \\
\hline & $\begin{array}{l}1.3 . \\
\text { Maleabilidad de } \\
\text { conocimientos } \\
\text { gramaticales y } \\
\text { formales de } \\
\text { CPL1 en } \\
\text { contextos y } \\
\text { situaciones } \\
\text { diferentes }\end{array}$ & $\begin{array}{l}\text { 1.3.1. Selecciona conocimientos gramaticales que son } \\
\text { relevantes para el contexto comunicativo solicitado. } \\
\text { 1.3.2. Omite conocimientos gramaticales que no son } \\
\text { relevantes para el contexto comunicativo solicitado. }\end{array}$ \\
\hline & $\begin{array}{l}1.4 . \\
\text { Maleabilidad de } \\
\text { conocimientos } \\
\text { prácticos previos } \\
\text { en contextos } \\
\text { diferentes, } \\
\text { mediante un } \\
\text { proceso de } \\
\text { selección y } \\
\text { descarte }\end{array}$ & $\begin{array}{l}\text { 1.4.1. Desarrolla ideas complejas de manera formal } \\
\text { 1.4.2. Elabora ideas descriptivas por medio de las } \\
\text { estrategias de redacción (definición, ejemplificación, } \\
\text { aclaración y causalidad) que interactúan con su postura } \\
\text { hacia un dilema ético o situación comunicativa } \\
\text { solicitada. } \\
\text { 1.4.3. Selecciona las estrategias para explicar su } \\
\text { postura. }\end{array}$ \\
\hline
\end{tabular}

Elaboración propia 
Ahora bien, sobre el instrumento que surge de esta operacionalización, podemos indicar lo siguiente: se establecen 4 niveles para lograr la transferencia. A su vez, tenemos 2 subcategorías vinculadas al tipo de logro que implica la transferencia de la expresión escrita. El primero es interpretación de conocimientos teóricos, es decir, temas relacionados a puntuación, léxico, ortografía y construcción oracional. La segunda subcategoría es la interpretación de conocimientos prácticos. Esta consiste en el logro de descripciones y argumentaciones mediante estrategias de redacción que permitan sustentar y fundamentar las ideas planteadas.

Tras esto, se establecen 2 niveles superiores: maleabilidad de conocimientos teóricos y la maleabilidad de conocimientos prácticos. Ambos, tanto en el plano técnico como en el plano analítico y relacionado con la sustentación de ideas, ameritan análisis, maleabilidad y aprehensión de un conocimiento previo, es decir, una activación de la transferencia de la expresión escrita.

\section{Análisis y resultados}

Encontramos una relación directa entre lo que mencionaba la teoría de la transferencia y los resultados de la indagación. Los alumnos lograron los niveles esperados de transferencia de la expresión escrita cuando ocurría uno de estos dos factores: o necesitaba comunicarse o le interesaba que lo entiendan. Esto implica que, en los espacios donde no existían esos dos aspectos, simplemente no se activaba la transferencia. Hemos identificado que los apartados que donde se daba esto eran en los de contenido y los de construcción oracional, ya que, si existe error en estos elementos, no se puede lograr una comprensión del texto. Por el contrario, esto no se ha dado en tal nivel en el uso de conectores y en aspectos más técnicos como la puntuación y ortografía, ya que, aun existiendo el error, se tiene la noción de que igual se puede, en cierta medida, entender el texto. En síntesis, y tras la codificación de resultados y de informantes, podemos observar lo siguiente.

En primer lugar, sobre los aspectos teóricos aprendidos en el curso de lenguaje, podemos indicar que es la fase en la que se evidenció un menor rendimiento. Esto se debe a que los errores, en su mayoría, fueron de puntuación, ortografía, pero que no dificultó la lectura y comprensión, es decir, no se activó ni la necesidad ni el interés en el proceso de redacción. Un ejemplo es el trabajo de la informante Karola:

En la cultura moche se utilizaban objetos para llevar a cabo sus rituales para la fertilidad. Actualmente la creencia de que estos objetos poseen propiedades mágicas sigue estando vigente, ya que estos se siguen utilizando en rituales de curandería hasta el día de hoy.

En esta cita se aprecia la relación que existe entre la cultura moche y los rituales realizados por chamanes, los cuales forman gran parte del turismo místico en la ciudad de Trujillo.

En este artículo se trata la producción utilitaria y ritual moche, en el sitio de Huacas de Moche, el cual se ubica en el norte del Perú (A7C15).

Podemos observar cómo constante el error del circunstancial de lugar al inicio de cada párrafo. Sin embargo, este tipo de error, si bien es repetitivo, no interrumpe o confunde en la lectura. Como resultado de análisis de este apartado, resumimos los resultados en el siguiente cuadro: 


\begin{tabular}{|l|l|l|l|l|l|l|}
\hline \multicolumn{1}{|c|}{ Estudiante/fase } & $\begin{array}{c}\text { Alberto } \\
\text { final }\end{array}$ & Karina final & $\begin{array}{c}\text { Karlota } \\
\text { final }\end{array}$ & $\begin{array}{c}\text { Karola } \\
\text { final }\end{array}$ & Katty final & Ketty final \\
\hline Categorías & & & & & & \\
\hline $\begin{array}{l}\text { Interpretación del } \\
\text { conocimiento } \\
\text { teórico aprendido } \\
\text { en el curso de } \\
\text { lenguaje. }\end{array}$ & & & & & & \\
\hline
\end{tabular}

Figura 1. Nivel logrado de cada estudiante, redacción parcial y final en lo que respecta a la primera subcategoría. Elaboración propia.

\begin{tabular}{|l|l|}
\hline Nominalización & Color \\
\hline Transferencia Nivel 1 & \\
\hline Transferencia Nivel 2 & \\
\hline Transferencia Nivel 3 & \\
\hline Transferencia Nivel 4 & \\
\hline
\end{tabular}

Figura 2. Leyenda para el nivel de logro de la transferencia. Elaboración propia.

Como podemos ver, los resultados son similares en todos los estudiantes. Lo importante no es tanto esa constante, sino el motivo de los resultados: mientras menor interés o necesidad por comunicarse, mayor cantidad de errores y menor necesidad para transferir aprendizajes previos, específicamente de cursos de redacción.

Caso contrario ocurre en el momento más básico de redacción de aspectos prácticos: en la interpretación del conocimiento práctico aprendido en el curso de lenguaje se evidencia una capacidad para desarrollar y plantear ideas principales y para comunicarse de manera efectiva. Esto lo podemos observar en una parte de la redacción de la alumna Katy, quien logra elaborar una adecuada reformulación por la necesidad esencial que tiene esta para la explicación de su problema de investigación:

Rousseau (1990), define a la cultura como conjunto de cogniciones compartidas por los miembros de una determinada unidad social, las cuales se adquieren a través del aprendizaje social y de procesos de socialización que expone a los individuos a diversos elementos culturales, como actividades e interacciones, informaciones comunicadas y artefactos materiales, que conforman la experiencia social al trabajo que dotan a sus miembro de valores compartidos, marcos de comprensión comunes y sistemas de creencias y de expectativas (A11C29).

Esto se convierte en una constante y, si bien no todos logran el máximo nivel de transferencia, se observa que, por la importancia que tiene el expresar ideas y parafrasear y vincular autores para sustentar y plantear las problemáticas de sus trabajos, se active la transferencia de lo aprendido en el curso de lenguaje. A continuación, mostramos la gráfica: 


\begin{tabular}{|l|l|l|l|l|l|l|}
\hline \multicolumn{1}{|c|}{ Estudiante/fase } & $\begin{array}{c}\text { Alberto } \\
\text { final }\end{array}$ & Karina final & $\begin{array}{c}\text { Karlota } \\
\text { final }\end{array}$ & $\begin{array}{c}\text { Karola } \\
\text { final }\end{array}$ & Katty final & Ketty final \\
\hline Categorías & & & & & & \\
\hline $\begin{array}{l}\text { Interpretación del } \\
\text { conocimiento } \\
\text { práctico aprendido } \\
\text { en el curso de } \\
\text { lenguaje. }\end{array}$ & & & & & & \\
\hline
\end{tabular}

Figura 3. Nivel logrado de cada estudiante, redacción parcial y final en lo que respecta a la segunda subcategoría. elaboración propia.

\section{Discusión de resultados}

Recordando lo planteado por Szulanski (1996), y Nonaka y Takeuchi (1995), la transferencia implica pasar de un conocimiento tácito a uno explícito de manera cíclica. De esta manera, se logra integrar aspectos teóricos con elementos prácticos para poder transferir conocimientos. Esto, si lo relacionamos con la teoría educativa constructivista, veremos que la transferencia puede existir como un modelo de evaluación por competencias, ya que nos permite ir más allá del curso y concentrarnos en un desarrollo holístico e integral de una competencia (Bloom, 2007). Ahora bien, en lo concerniente a esta investigación, nos centramos en el logro o no de la transferencia de la expresión escrita. A nivel teórico, evidenciamos la falta de una reflexión más compleja de las fuentes en relación con lo que implica el lenguaje y el acto de expresarse (Wittgenstein, 2013). A partir de esto, logramos identificar el denominado stickiness por Szulanski (1996), es decir, aquello que impide el avance; que imposibilita el giro cíclico entre lo teórico y lo práctico. En el caso de la expresión escrita, el stickiness es la falta de activación del interés o necesidad por comunicarse.

El interés y la necesidad deben estar presentes para poder comprender el logro o no de la transferencia de la expresión escrita. A su vez, se debe comprender a cabalidad el fenómeno. Las investigaciones encontradas nos mostraron una falta de interés por la temporalidad y la recontextualización del saber, lo cual se determina en la decisión por quedarse en el curso y no centrarse en la competencia en sí. De este modo, las indagaciones previas tienen dos falencias: no se alejan ni contextual ni temporal ni a nivel de contenido del aprendizaje primigenio (se desarrollan en el mismo ciclo; no de forma posterior). Por ello, no se puede comprender a cabalidad el desarrollo de la competencia. Esta complicación en la comprensión de la competencia se puede solucionar desde lo realizado en este trabajo: la transferencia como modelo evaluativo.

Tras la aplicación del análisis de contenido y mediante el instrumento para analizar la transferencia de la expresión escrita, hemos encontrado una relación directa entre la teoría y la práctica. Ante la necesidad o el interés por expresar sus ideas, los estudiantes activaron conocimientos aprendidos en ciclos anteriores. Esto se generó en los aspectos vinculados al análisis y a aquellos en los que, si la redacción tenía falencias, el lector no comprenderían lo que quería decir. Caso contrario ocurre en los aspectos más técnicos. Los alumnos no vieron la necesidad de transferir aprendizajes previos, ya que identificaron que este tipo de errores no evitaban la comprensión del texto.

\section{Conclusiones}

La capacidad de los estudiantes para desarrollar procesos de transferencia de los conocimientos aprendidos de cursos de redacción en contextos posteriores está supeditada al interés y necesidad de 
enunciarse de modo escrito en el nuevo contexto comunicativo. Esto se debe a que el alumno evidencia que debe realizar una tarea comunicativa de manera óptima, ya que, de no hacerlo, no se comprenderá lo que deseó enunciar. Por el contrario, no se comunica de manera adecuada cuando identifica que sus errores podrán ser subsanados en la lectura de su redacción.

La capacidad para desarrollar procesos de interpretación del conocimiento teórico aprendido de cursos de redacción en contextos posteriores no se logró porque no existía la necesidad ni el interés. Esto es porque los alumnos identificaron que sus textos eran comprensibles, así como se encontraban y porque no el producto a elaborar no era de su completo interés.

La capacidad de los estudiantes para desarrollar procesos de interpretación del conocimiento práctico aprendido de cursos de redacción en contextos posteriores se logró cuando se presentó el interés y necesidad por parte del aquel. Cuando el alumno identificó que, de no redactar bien ciertos aspectos, no se comprendería lo que deseaba enunciar, se activó, en parte, la transferencia. A su vez, esto ocurrió cuando lo redactado era del interés de aquel.

En los textos evaluados, la capacidad de maleabilidad de aspectos teóricos de lenguaje por parte de los estudiantes de Metodología de la Investigación se produce cuando el estudiante tiene interés para desarrollar un texto formal y la necesidad de su emisión para la comprensión de la investigación que está desarrollando. Esto se produjo, especialmente, cuando el estudiante identificó que si no se comunicaba de forma correcta no sería entendido por su interlocutor (en este caso, el docente del curso).

\section{Referencias}

Alt-Gehrman, P. (2019). Nursing simulation and transfer of knowledge in undergraduate nursing programs: A literature review. Nursing Education Perspectives, 40(2), 95-98. doi: 10.1097/01.NEP.0000000000000398

Álvarez, O. H., y Ramírez, D. A.S. (2012). ¿Cómo enseñar el área de lenguaje con un enfoque constructivista, utilizando en el aula un computador, un videoproyector e internet? Revista Educación $\quad y \quad$ Pedagogía, 24, 219-230. Recuperado de https://revistas.udea.edu.co/index.php/revistaeyp/article/view/14205

Arias, Ó., y Collazos, J. (2015). Knowledge management in research groups of higher education institutions in Colombia. En A. Garlatti y M. Massaro (Ed.), Proceedings of the 16 European Conference on Knowledge Management: ECKM 3-4 Sep. (pp. 47-55). Udine, Italia: Academic Conferences and Publishing International Limited. Recuperado de https://docplayer.net/47270315-16th-european-conference-on-knowledge-managementeckm-2015.html

Ausubel, Novak, y Hanesian (1983). Psicología educativa. Un punto de vista cognoscitivo. México: Trillas. Austin, J.L. (1998) Cómo hacer cosas con palabras. México: Siglo XXI.

Badea, A., Prostean, G., Adam, A., y Giuca, O. (2013). Knowledge management and creative thinking framework integrated in training of future students. Proceedings of the 14th European Conference on Knowledge Management: ECKM 5-6 Sep. (pp. 825-832). Kaunas, Lituania: Academic Conferences and Publishing International Limited. Recuperado de http://toc.proceedings.com/19357webtoc.pdf

Becheikh, N., Ziam, S., Idrissi, O., Castonguay, Y., y Landry, R. (2010). How to improve knowledge transfer strategies and practices in education? Answers from a systematic literature review. Research in Higher Education Journal, 7, 1-21. Recuperado de http://www.aabri.com/manuscripts/09418.pdf

Blackman, D., y Benson, A. (2010). Overcoming knowledge stickiness in scientific knowledge transfer. Public Understanding of Science, 21(5), 573 - 589. doi: 10.1177/0963662510379463

Bloom, J. (2007). Atheoretical model of learning and complexity: Depth, extent, abstraction and transfer. Anual Meeting of the American Educational Research Association. Chicago, EE.UU. 
Buissink-Smith, N., Mann, S., y Shephard, K. (2011). How Do We Measure Affective Learning in Higher Education? Journal of Education for Sustainable Development, 5(1), 101-114. doi: $10.1177 / 097340821000500113$

Carpintero, E., y Beltrán, J. (2010). Medida de transfer en el entorno educativo: resultados de la aplicación de la prueba PEPT. Psicothema, 22(3), 449-454. Recuperado de http://www.psicothema.com/psicothema.asp?id=3751

Cassany, D. (1990) Enfoques didácticos para la enseñanza de la expresión escrita. Comunicación, lenguaje y educación, 6, 63-80. Recuperado de http://www.upf.edu/pdi/dtf/daniel_cassany/enfoqes.htm

Cassany, D. (2012). La metamorfosis digital: cambios, ventajas y riesgos de leer y escribir en la red. En D. Goldin, M. Kriscautzky y F. Perelman. (Coord.), Las TIC en la escuela, nuevas herramientas para viejos y nuevos problemas. (pp. 217-236). Océano Travesía.

Cisneros-Estupiñán, M., Olave-Arias, G., y Rojas-García, I. (2012). Cómo mejorar la capacidad inferencial en estudiantes universitarios. Educación y Educadores, 15(1), 45-61. Recuperado de https://educacionyeducadores.unisabana.edu.co/index.php/eye/article/view/2130

Collins, S. (2012). The emotional intelligence profiles and cognitive measures of nurse anesthesia students in the southeastern united states (Tesis de doctorado). Universidad de Andrews, Michigan, EE.UU.

Crochet, P., Agostini, A., Knight, S., Resseguier, N., Berdah, S., y Aggarwal, R. (2017). The performance gap for residents in transfer of intracorporeal suturing skills from box trainer to operating room. Journal of Surgical Education, 74(6), 1019-1027. doi: 10.1016/j.jsurg.2017.05.013

de Castro, J. M., Daniela, M. D., Roberto, G. D., Dressler, M., y Rodrigo Baroni, d. C. (2013). Critical determinants within knowledge transfer processes: A case study at embrapa. Revista De Administração Pública, 47(5), 1283-1306. doi: 10.1590/S0034-76122013000500010

Lazo, D. (2017). Análisis de los procesos de transferencia de conocimientos de lenguaje en contextos de aprendizaje de cursos formativos (Tesis de maestría). Universidad Peruana Cayetano Heredia, Lima, Perú.

Makuc, M. (2008). Teorías implícitas de los profesores acerca de la comprensión de textos. Revista Signos, 41(68), 403-422. Recuperado de https://scielo.conicyt.cl/pdf/signos/v41n68/art03.pdf

Martínez-Díaz, E. S., Rodríguez, D.E., y Díaz, N. (2011). El andamiaje asistido en procesos de comprensión lectora en universitarios. Educación y Educadores, 14(3), 531-555. Recuperado de https://educacionyeducadores.unisabana.edu.co/index.php/eye/article/view/2044

Maynard, C. A. (1991). The relationship of critical thinking ability, stage of skill acquisition, years of practice, and organizational context to professional nursing competence (Tesis doctoral). Universidad de Minnesota, Minnesota, EE.UU.

Nonaka, I., y Takeuchi, H. (1995). The Knowledge-Creating Company. Oxford, EE. UU: Oxford University Press.

Piaget, J. (2001). Psicología y pedagogía. Barcelona, España: Planeta.

Szulanski, G. (1996). Exploring Internal Stickiness: Impediments to the Transfer of Best Practice within the Firm. Strategic Management Journal, 17, 27-43. doi: 10.1002/smj.4250171105

Trillos-Pacheco, J. (2013). La lectura hipermedial y su incidencia en la comprensión lectora en estudiantes universitarios. Palabra Clave, 16(3), 944-992. Recuperado de http://hdl.handle.net/11323/4801

Van Dijk, T. y Kintsch, W. (1983). Strategies of Discourse Comprehension. Nueva York, EE. UU: Academic Press.

Von Hippel, E. (1994). "Sticky Information" and the locus of Problem Solving: Implications for Innovation. Management Science, 40(4), 429-439. doi: 10.1287/mnsc.40.4.429

Wittgenstein, L. (2013). Tratactus Logico-philosophicus. Madrid, España: Tecnos. 